\title{
Monitoring of Bright Blazars with MAGIC in the 2007/2008 Season
}

\author{
Konstancja Satalecka ${ }^{\star, 1}$ Ching-Cheng Hsu, ${ }^{\star, 2}$ Elisa Bernardini, ${ }^{1}$ \\ Giacomo Bonnoli ${ }^{3}$ Nicola Galante, ${ }^{2}$ Florian Goebel, ${ }^{2, \dagger}$ Elina Lindfors, ${ }^{4}$ \\ Pratik Majumdar, ${ }^{1}$ Antonio Stamerra ${ }^{3}$ and Robert Wagner ${ }^{2}$ \\ on behalf of the MAGIC collaboration \\ ${ }^{1}$ DESY, Platanenallee 6, D-15738 Zeuthen, Germany, \\ ${ }^{2}$ Max-Planck-Institut für Physik, Föhringer Ring 6, D-80805 München, Germany, \\ ${ }^{3}$ Dipartimento di Fisica, Università degli Studi di Siena, Via Roma 56, I-53100 Siena, Italy and \\ ${ }^{4}$ Tuorla Observatory, Dept. Physics and Astronomy, University of Turku, FI-20014 Turku, Finland. \\ *Corresponding authors.Email: konstancja.satalecka@desy.de, cchsu@mppmu.mpg.de \\ $\dagger$ deceased
}

\begin{abstract}
Because of the short duty-cycles and observation-time constraints, studies of bright $\mathrm{TeV}$ (E>100 GeV) blazars are mostly restricted to flaring episodes or rather short (days to few weeks) multiwavelength campaigns. At the same time, long-term studies of these objects are essential to gain a more complete understanding of the blazar phenomenon and to constrain theoretical models concerning jet physics. Only unbiased long-term studies are adequate for the determination of flaring state probabilities and for estimating the statistical significance of possible correlations between $\mathrm{TeV}$ flaring states and other wavebands or observables, such as neutrino events. Regular observations also provide triggers for multiwavelength $\mathrm{ToO}$ observations originating from the $\mathrm{TeV}$ waveband. These are particularly needed to identify and study orphan $\mathrm{TeV}$ flares, i.e. flares without counterparts in other wavebands. In 2007/8 the MAGIC telescope has monitored three TeV blazars on a regular basis: Mrk 501, Mrk 421, and 1ES 1959+650. We present preliminary results of these observations including the measured light curves and a correlation study for VHE $\gamma$-rays and X-rays and VHE $\gamma$-rays and optical R-band for Mrk 421.
\end{abstract}

Keywords: Active Galactic Nuclei: individual; BL Lacertae objects: individual; gamma-rays: observations; gamma-ray telescopes

PACS: $95.55 . \mathrm{Ka}, 95.85 . \mathrm{Pw}, 98.54 . \mathrm{Cm}$

\section{INTRODUCTION}

With the new generation of Imaging Atmospheric Cerenkov Telescopes (IACTs) like HESS [1], MAGIC [2] and VERITAS [3] Very High Energy (VHE) $\gamma$-ray astronomy became a very dynamic and exciting discipline with many new detections of galactic and extra-galactic objects every year. Almost one third of the objects detected in VHE $\gamma$-rays are blazars, i.e. Active Galactic Nuclei (AGNs) which contain relativistic jets pointing approximately in the direction of the observer. Their energy spectra show no or very weak emission lines, but a continuous distribution with two broad peaks: one in the UV to soft X-ray band and a second one in the GeV-TeV range. One of the most interesting aspects of blazars is their variability, observed in all frequencies and on different time scales ranging from weeks to minutes [4] [5].

The data collected so far in many multiwavelenght observations is not yet enough 
to fully constrain the theoretical models which try to explain the acceleration and emission mechanisms in blazars. In particular it is not yet clear if the leptonic or hadronic processes play a decisive role. For example, the Synchrotron-Self Compton (SSC) leptonic models [6] can successfully describe most of the existing data and offer a reasonable explanation of the fast variability of blazars. On the other hand, the hadronic models, like the Synchrotron Mirror Model (SMM) [7] or Synchrotron Proton Blazar (SPB) [8] model can also very well describe the SED structure. Additionally they explain the "orphan" gamma-ray flares (like the one observed for 1ES 1959+650 in 2002 [9]) and predict emission of high energy neutrinos.

\section{AGN MONITORING}

A long term monitoring of the VHE $\gamma$-ray flux variability performed in the GeV-TeV energy range will give vital input to understand the problems mentioned above.

Observations scheduled independently of any knowledge of the source state provide us with an unbiased distribution of the flux states. Many of the previously performed measurements were triggered by an observed enhancement of flux state in other wavebands and therefore observations of AGNs during a low flux state are still sparse. Consequently any statistical study which requires high statistics on various flux levels is difficult. An example of such a study might be the determination of flaring state probabilities, essential for estimation of the statistical significance of possible correlations between flaring states and other observables, such as neutrino events [10]. Here a long term monitoring plays a crucial role, especially in a view of the results expected from the IceCube neutrino observatory [11].

Another interesting application is the investigation of spectral changes during periods of different source activity, which can improve our knowledge about the acceleration and emission processes.

Last but not least AGN monitoring can serve to trigger Target of Opportunity (ToO) observations. The ToO observations may be performed by the IACT issuing the ToO trigger but may also include other IACTs - allowing to increase the time coverage of the observations - or telescopes and satellites observing other wavelengths. X-ray obsrvations are especially interesting in a context of the "orphan" TeV flares.

\section{THE MAGIC TELESCOPE}

MAGIC is currently the largest single-dish IACT for VHE $\gamma$-ray astronomy. It is located on the Canary Island of La Palma, at an altitude of $2200 \mathrm{~m}$ a.s.l and has been in scientific operation since summer 2004. The camera is equipped with 576 photo-multiplier tubes (PMTs). In January 2007 a major upgrade of the MAGIC Telescope with a Multiplexed Fiber-Optic 2 GSamples/s FADC Data Acquisition system took place [12]. The fast readout minimizes the influence of the background from the light of the night sky and the additional information on the time structure of the event helps to reduce the background from hadronic showers [13]. The trigger threshold of MAGIC is around 


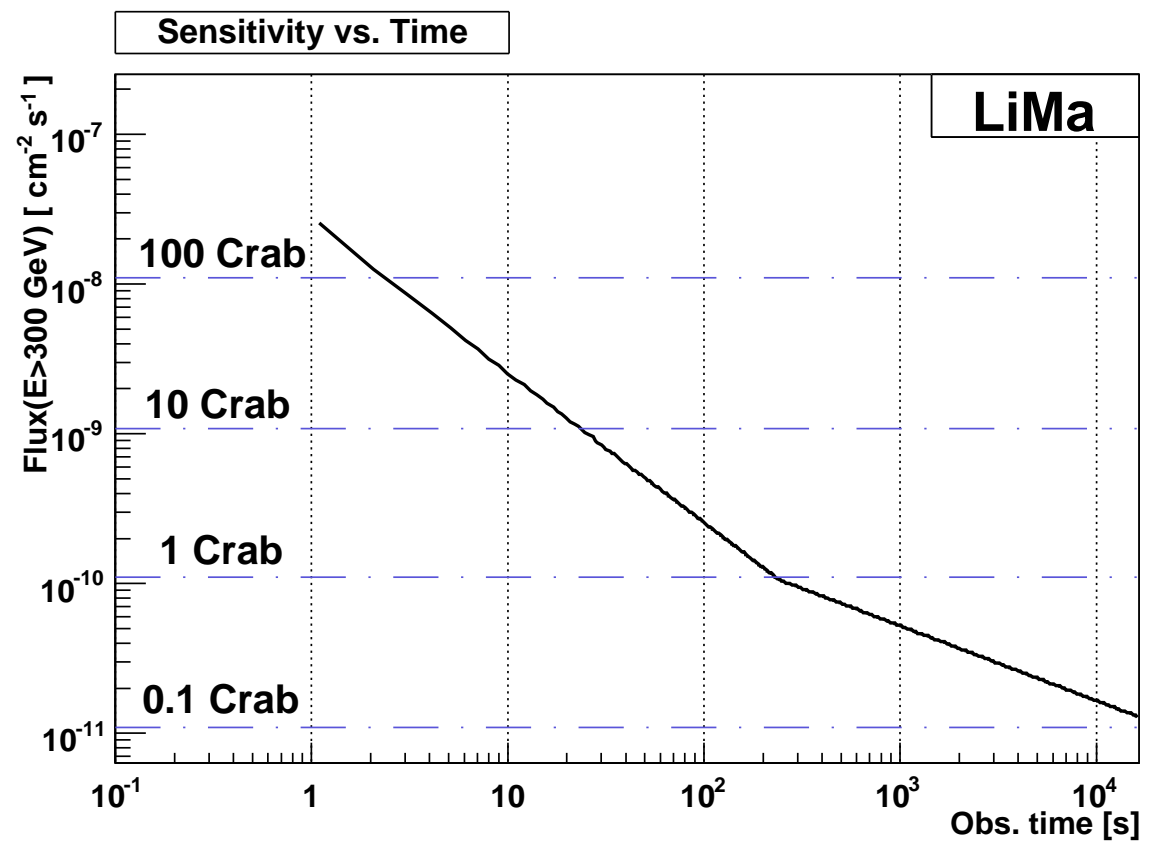

FIGURE 1. MAGIC sensitivity [14] as a function of exposure time.

$60 \mathrm{GeV} 1$. A source emitting $\gamma$-rays at a flux level of $1.6 \%$ of the Crab Nebula can be detected with $5 \sigma$ significance within 50 hours of observation time. The sensitivity is sufficient to establish a flux level of about $30 \%$ of the Crab flux above $300 \mathrm{GeV}$ for a 30 min observation (Fig.1). A quick on-line analysis system allows to estimate the flux level of the observed source and facilitate the issuing of ToO triggers during data taking. MAGIC can also observe under moderate moon and twilight conditions with only slightly lower sensitivity. The construction of a second telescope (MAGIC-II) is being finalized and the new telescope is undergoing various tests. It is planned to start stereoscopic observations in spring 2009.

\section{MONITORING STRATEGY}

Due to the low duty cycle $(10-12 \%)$ the operation time of IACTs is very precious and the observations have to be scheduled very carefully in order to achieve the right balance between the high priority, deep observations and AGN monitoring. Therefore usually previous generation IACTs which are still operational are used for this purpose. For example the Whipple ${ }^{2}$ telescope, which is leading a monitoring program since 2005 for five well established TeV sources, or DWARF, one of the former HEGRA telescopes currently being refurbished and brought back into operation on La Palma [15]. In the

\footnotetext{
1 The trigger threshold is defined as the peak of the energy distribution of the triggered events.

$2 \mathrm{http}: / /$ veritas.sao.arizona.edu/content/blogsection/6/40/
} 
case of MAGIC, a high sensitivity latest generation IACT, some of the monitoring observations can be performed during moderate moonlight or twilight, which keeps the impact on the overall observation schedule low.

In order to achieve a dense sampling, up to 40 short observations per source are scheduled, evenly distributed over the observable time during MAGIC observation period. Each of them should be long enough to detect a given minimum flux level taking into account the sensitivity of the telescope. In the case of MAGIC, three sources were chosen for a regular monitoring: Mrk 421, Mrk 501 and 1ES 1959+650. The first two are relatively bright and usually 15-30 min observations are scheduled for them. 1ES 1959+650 being fainter requires longer observation times, at least 30 minutes per single exposure.

\section{RESULTS}

In this section we will present the preliminary results of the MAGIC AGN monitoring program for the observation season 2007/2008. The data have been processed with the standard MAGIC analysis tools [2]. A fraction of the data has been removed due to poor observation conditions. All cuts are optimized and verified with Crab data in the corresponding observational seasons.

\section{Mrk 421}

Mrk 421 is the first extragalactic object detected at energies above $500 \mathrm{GeV}$ [16] [17] and one of the best studied. Here the preliminary results of the data which were taken from February 2007 to June 2008 are presented (82 hours). After the data selection, about 66 hours of data $(80 \%)$ were used for further analysis. The observations were mostly performed in wobble mode, which allows to simultaneously collect signal and background events.

Fig. 2 shows all the data collected from Mrk 421 by the MAGIC telescope since 2004 [18] [19]. In Fig 3 the 2007/2008 observational period is shown magnified. We can clearly see that Mrk 421 was especially active in 2008 and the flux was never below 0.5 Crab. During this time of high activity, in accordance with the ToO agreement, MAGIC issued several alerts to HESS and VERITAS. Detailed analysis of the collected data, such as intra-night variability and spectrum measurement will be published elsewhere.

In the SSC framework, a correlation between $\mathrm{TeV}$ photon and the other wavelengths, especially X-ray, is expected [20]. Since usually the TeV data is relatively poorly sampled, it is not easy to get really simultaneous data, we therefore searched for X-ray and optical measurements which are within $6 \mathrm{~h}$ before or after the TeV observations.

In this work we use the X-ray data taken by the RXTE/ASM satellite, which is designed for all sky monitoring observations. The data are available on the web page immediately after the observations. The averages and errors of ASM data points are

3 http://xte.mit.edu/ASM_lc.html 


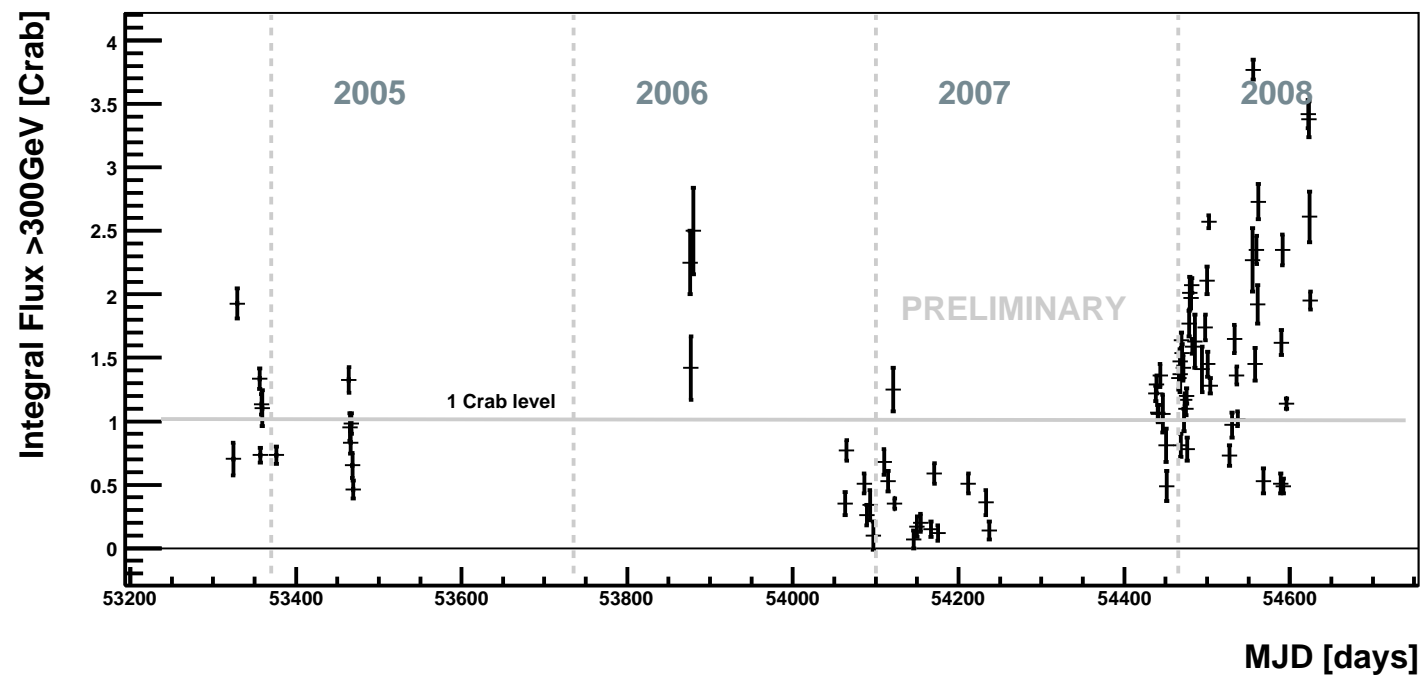

FIGURE 2. Mrk 421 light curve showing all data collected by MAGIC [18] [19].

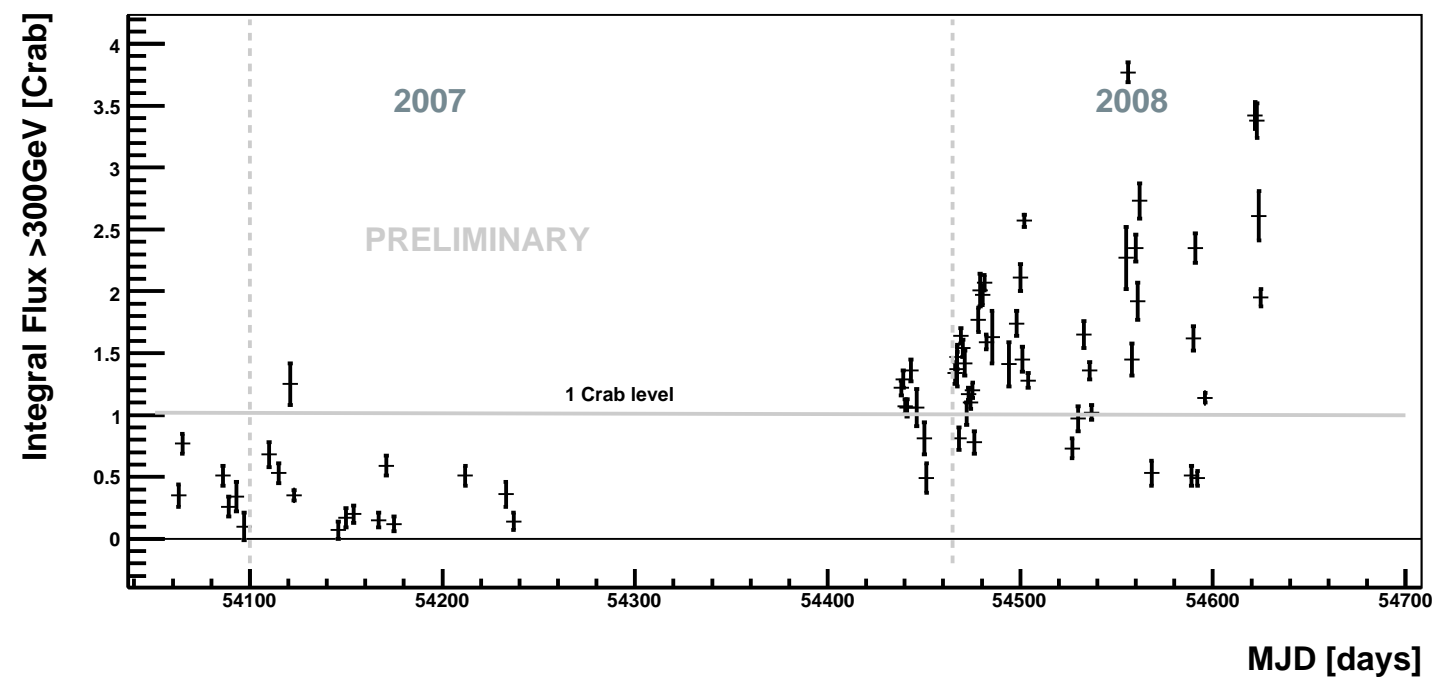

FIGURE 3. Mrk 421 light curve showing MAGIC data from AGN monitoring season 2007/2008.

calculated on a dwell-by-dwell 14 data basis. If the number of dwells are fewer than five, we discard that data point; $75 \mathrm{TeV}$-X-ray measurements pairs were finally selected (shown in Fig,4).

The optical R-band data were provided by the Tuorla Observatory Blazar Monitoring Program 5 with the $1.03 \mathrm{~m}$ telescope at the Tuorla Observatory Finland and the $35 \mathrm{~cm}$ KVA telescope at La Palma, Canary Islands. From the optical flux the flux of the host

4 one dwell lasts 90 seconds

${ }^{5}$ http://users.utu.fi/kani/1m/ 


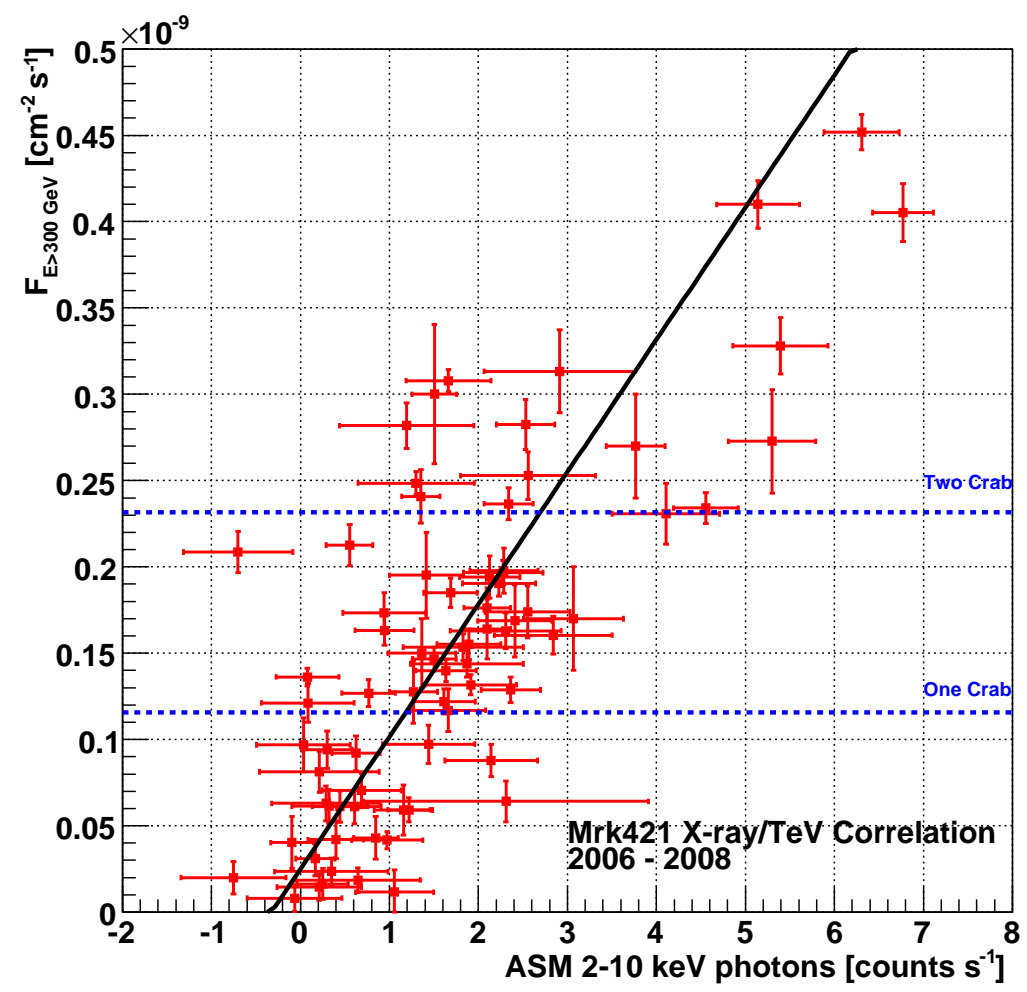

FIGURE 4. VHE $\gamma$-ray (MAGIC) - X-ray (ASM) correlation plot for Mrk 421.

galaxy and the flux contribution from the companion galaxies were substracted [21]. Eventually, 56 measurements pairs were found (Fig.5).

The correlation coefficients for both plots were calculated using Pearson's method (see section 14.5 in [22]). For X-ray/TeV correlation, the correlation coefficient value is $r=0.77 \pm 0.05$. For optical/TeV plot, the coefficient value is $r=0.03 \pm 0.14$. We thus found a significant correlation with X-rays. This type of correlation is easier accommodated in leptonic models (see e.g. [23] and references therein) but there also exist certain hadronic models which support such correlation (see e.g. [8] or [24]). However, one has to keep in mind, that the data were not taken strictly simultaneously and any conclusion as to the origin or mechanisms of the emission should be made very carefuly.

\section{Mrk 501}

The BL Lac object Mrk 501 was the second established TeV-blazar [25] [26]. MAGIC has been observing this source since the year 2005 [5]. Here we present preliminary results based on an observation time of 16 hours (after quality selection), collected between February 2007 and August 2008. In order to maximize the time coverage of this source, observations were carried out mostly in the presence of moderate moonlight 


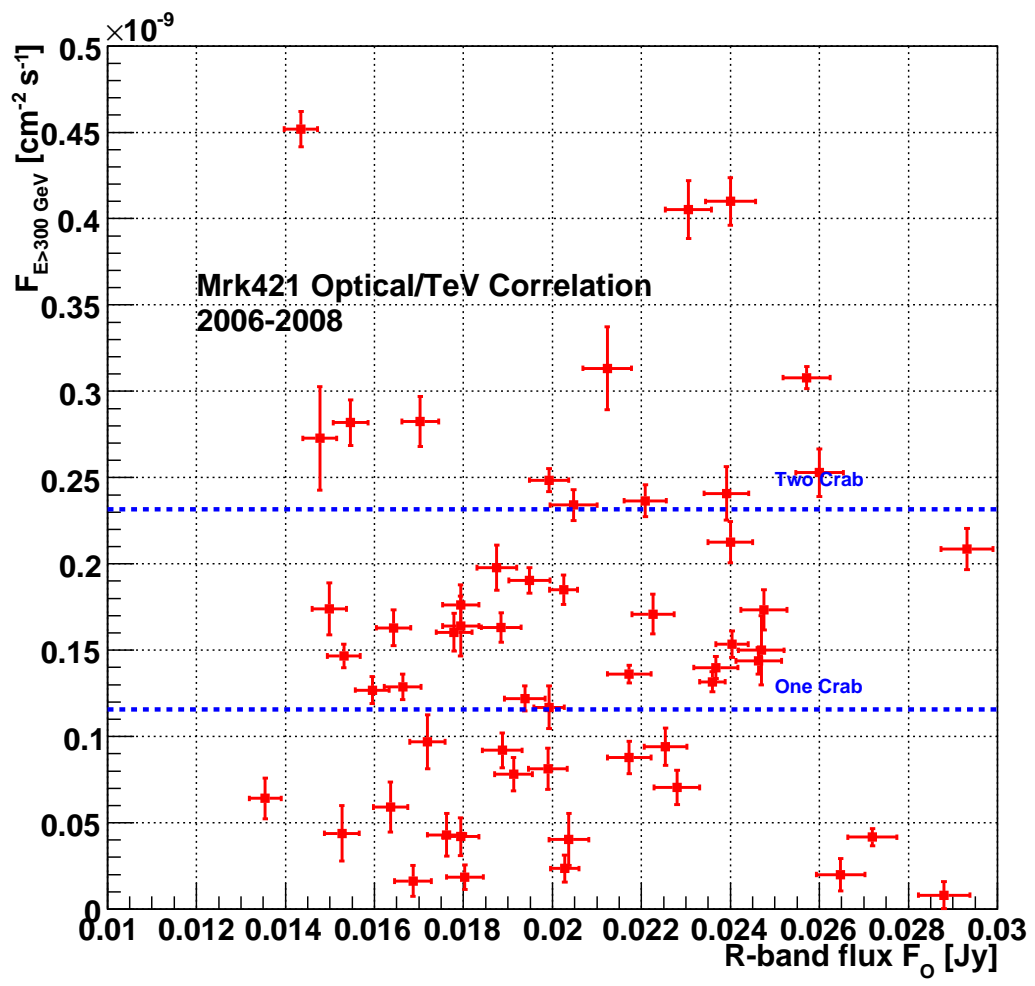

FIGURE 5. VHE $\gamma$-ray (MAGIC) - R-band (Tuorla) correlation plot for Mrk 421.

or twilight ( 9 hours, i.e. $56 \%$ of the observation time). The observations were performed in wobble mode.

Fig. 6 shows all the data collected form Mrk 501 by the MAGIC telescope since 2005 [5] [19]. In Fig. 7 the 2007/2008 monitoring season is shown magnified. During this period the source was in a relatively low state (below $1 \mathrm{Crab}$ ). On the other hand a dense sampling was obtained and a statistical analysis, e.g. estimation of the of the source state probability, based on the above data is in progress.

\section{ES 1959+650}

The observation of an "orphan" TeV flare (i.e. not accompanied by a corresponding X-ray flare) in 2002 [9] made 1ES 1959+650 one of the most interesting VHE $\gamma$-ray sources. MAGIC has been observing this object since 2004 [27], a regular monitoring was first performed in the 2005/2006 season [19]. Unfortunately, the source position allows observations only under a relatively high zenith angles (35-50 deg). Moreover, when not in a high state the source is rather faint compared to those discussed above.

MAGIC observed 1ES 1959+650 from April 2007 till October 2008 for 27 hours (including 13 hours of data taken during moonlight or twilight). Observations were 


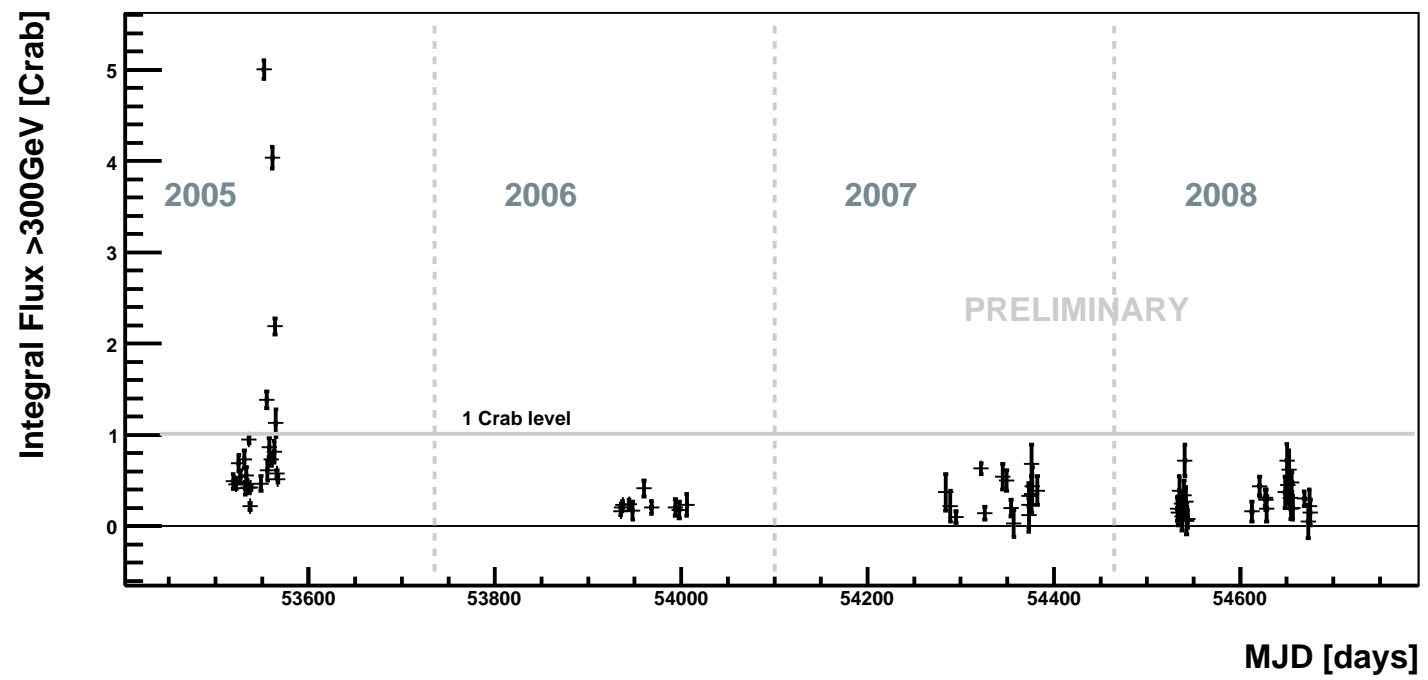

FIGURE 6. Mrk 501 light curve showing all data collected by MAGIC [19] [5].

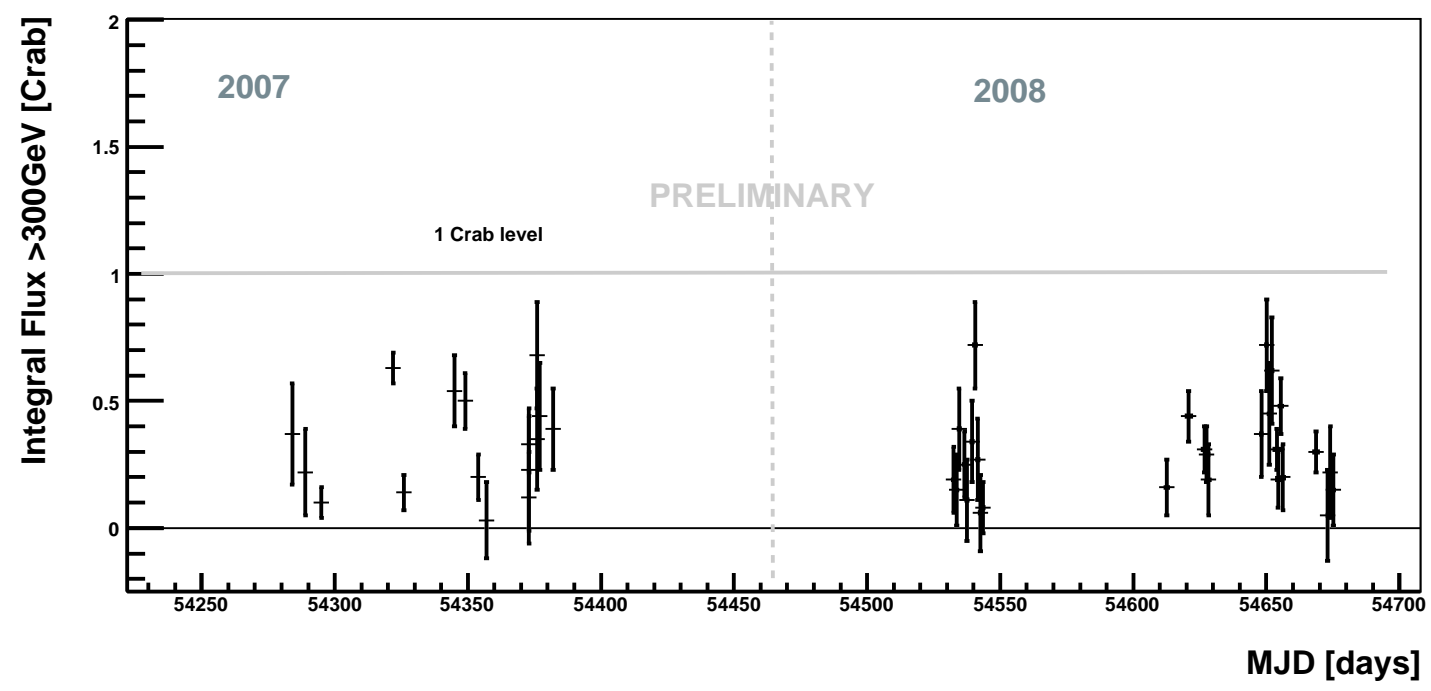

FIGURE 7. Mrk 501 light curve showing MAGIC data from AGN monitoring season 2007/2008.

performed in wobble mode. After rejection of data due to poor quality 3.4 hours of data taken in 2008 were analyzed. The overall significance of this sample is $2.5 \sigma$, which allows us to set an upper limit on the flux above $300 \mathrm{GeV}$ of $2.53 \times 10^{-11} \mathrm{ph} / \mathrm{cm}^{2} / \mathrm{s}$ at 90\% C.L. Comparing to previous results [28] [29] we can state that MAGIC observed this source in its usual low state. 


\section{CONCLUSIONS}

During the observational season 2007/2008 MAGIC monitored three sources: Mrk 421, Mrk 501 and 1ES 1959+650. The detailed analysis of the collected data is going on. In this work we presented preliminary results of the measured flux levels for all three sources. We also investigated the possible correlation of the $\mathrm{TeV}$ and $\mathrm{X}$-ray/optical flux levels for Mrk 421. We found a significant correlation with X-rays and no correlation with optical R-band. The most interesting material for further studies are the many fast flares observed for Mrk 421 in 2008. Mrk 501 and 1ES 1959+650 were found in low state, but dense sampling of Mrk 501 flux states looks promising for statistical studies.

\section{ACKNOWLEDGMENTS}

We would like to thank the Instituto de Astrofisica de Canarias for the excellent working conditions at the Observatorio del Roque de los Muchachos in La Palma. The support of the German BMBF and MPG, the Italian INFN and Spanish MCINN is gratefully acknowledged. This work was also supported by ETH Research Grant TH 34/043, by the Polish MNiSzW Grant N N203 390834, and by the YIP of the Helmholtz Gemeinschaft.

\section{REFERENCES}

1. F. Aharonian, et al., Astron. Astrophys. 457, 899-915 (2006).

2. J. Albert, et al., Astrophys. J. 674, 1037-1055 (2008).

3. V. A. Acciari, et al., Astrophys. J. 679, 1427 (2008).

4. F. Aharonian, Astrophys. J. 664, L71 (2007).

5. J. Albert, et al., Astrophys. J. 669, 862 (2007).

6. F. Tavecchio, L. Maraschi, and G. Ghisellini, Astrophys. J. 509, 608-619 (1998).

7. A. Reimer, M. Bottcher, and S. Postnikov, Astrophys. J. 630, 186-190 (2005).

8. A. Muecke, et al., Astropart. Phys. 18, 593-613 (2003).

9. H. Krawczynski, et al., Astrophys. J. 601, 151-164 (2004).

10. K. Satalecka, et al., Contributions to the ICRC, Merida, Mexico, Jul. 3-11 pp. 115-118 (2007), 0711.0353 .

11. J. Ahrens, et al., Astropart. Phys. 20, 507-532 (2004).

12. F. Goebel, et al., Contributions to the ICRC, Merida, Mexico, Jul. 3-11 (2007), 0709.2363.

13. E. Aliu, et al., accepted by Astropart. Phys. (2008).

14. T. Li, and Y. Ma, Astrophys. J. 273, 317-324 (1983).

15. T. Bretz, M. Backes, I. Braun, et al., "Long-term monitoring of bright blazars with a dedicated Cherenkov telescope," in High Energy Gamma-Ray Astronomy, 2009, to be published.

16. M. Punch, et al., Nature 358, L477-L478 (1992).

17. D. Petry, et al., Astron. Astrophys. 311, L13-L16 (1996).

18. J. Albert, et al., Astrophys. J. 663, 125-138 (2007).

19. F. Goebel, et al., Contributions to the ICRC, Merida, Mexico, Jul. 3-11 (2007), 0709.2032.

20. L. Maraschi, et al., Astropart. Phys. 11, 189-192 (1999).

21. K. Nilsson, et al., Astron. Astrophys. 475, 199-207 (2007).

22. W. H. Press, et al., SIGSOFT Softw. Eng. Notes 28, 36-36 (2003), ISSN 0163-5948.

23. R. Wagner, PoS(BLAZARS2008)013 (2008).

24. F. A. Aharonian, New Astron. 5, 377-395 (2000).

25. J. Quinn, et al., Astrophys. J. 456, L83-L86 (1996).

26. S. M. Bradbury, et al., Astron. Astrophys. 320, L5-L8 (1997).

27. E. Aliu, et al., Astrophys. J. 639, 761-765 (2006). 
28. F. Aharonian, et al., Astron. Astrophys. 406, L9-L13 (2003).

29. K. Gutierrez, et al., Astrophys. J. 644, 742-747 (2006). 